\title{
Clinical Retrospective Analysis of Hospitalized Patients with Ocular Trauma
}

\author{
Liang Li Cheng ${ }^{1}$, Liang Liang ${ }^{2}$, and Li Qing Hua ${ }^{2}$ \\ ${ }^{1}$ Gezhouba senior high school, Yichang city, China \\ ${ }^{2}$ Department of ophthalmology, Yichang central hospital, Yichang city, China \\ *Corresponding author: Liang Liang, Department of ophthalmology, Yichang central hospital, Yichang city, China
}

\section{ARTICLE INFO}

Received: 幽 December 02, 2019

Published: 慧December 10, 2019

Citation: Liang Li Cheng, Liang Liang, Li Qing Hua. Clinical Retrospective Analysis of Hospitalized Patients with Ocular Trauma. Biomed J Sci \& Tech Res 23(4)-2019. BJSTR. MS.ID.003949.

Keywords: Ocular Trauma; Epidemiology; Related Factors

Abbreviatations: EIR: Eyes Injury Registry; USEIR: United States Eyes Injury Registry; NUH: National University Hospital; WHO: World Health Organization
ABSTRACT

Objective: To analyze the related factors of ocular trauma in ophthalmic inpatients and provide epidemiological data on local ocular trauma.

Methods: 1137 cases of 1610 eyes with ocular trauma admitted to our hospital from January 2014 to December 2018 were selected for statistical analysis of general conditions, causes of injury, prognosis and complications.

Results: The ratio of male to female was 4.77:1, and the peak age was 20 to 49 years old. The occupation was mainly manual workers such as workers and farmers. The first two injuries were blunt and perforated injuries, of which the average of perforation injuries the highest rate of blindness $(45.65 \%)$. There was no significant difference in the composition of injury between adjacent years in the past five years $(\chi 2=14.586, \mathrm{P}$ $=0.367$ ). Complications of ocular trauma are mixed, and traumatic cataracts, anterior chamber hemorrhage, and uveitis are more common.

Conclusion: People should improve their awareness of the prevention of ocular trauma. Patients with ocular trauma can be treated promptly to reduce the blindness rate of ocular trauma.

\section{Introduction}

Eye trauma is the world's first cause of blindness [1]. Epidemiological data from domestic and foreign scholars on ocular trauma have shown that ocular trauma patients are mainly concentrated in young people, which means that ocular trauma not only brings physical and mental harm to the patient, but also brings great family and society awkward medical burden and economic loss. Therefore, it is necessary for us to conduct extensive and extensive epidemiological investigations on ocular trauma to guide prevention and clinical treatment. At present, the Eyes Injury Registry (EIR) has been established in many countries including the United States [2]. In China, although ocular trauma has long been ranked as one of the three major blind diseases (cataract, glaucoma, and ocular trauma) in ophthalmology, it currently lacks epidemiological data on ocular trauma in the whole country and in some areas. Our hospital is located in Yichang, China, and belongs to the western part of Hubei province. The local transportation is convenient, the population density is high, the incidence of ocular trauma is relatively high, and local epidemiology of ocular trauma is rarely reported. Therefore, the ocular trauma patients admitted to the Department of Ophthalmology in our hospital from January 2014 to December 2018 were comprehensively analyzed and analyzed as follows to provide relevant information for the epidemiological investigation of ocular trauma in western Hubei province, China.

\section{Methods}

Subjects collected medical records of 4427 inpatients admitted to the Department of Ophthalmology, Yichang Central Hospital from January 2014 to December 2018. The first diagnosis was 1137 patients with ocular trauma, 1610 eyes, accounting for $25.68 \%$ 
to occupy the total number of hospitals in the same period of ophthalmology. Methods The general conditions (including gender, age distribution, occupational distribution), injury characteristics, prognosis visual acuity and complications of 1137 patients with 1610 eyes with ocular trauma were sorted out. Statistical analysis: $\chi 2$ test was performed on the count data using SPSS 17.0 statistical software, with $\mathrm{P}<0.05$ is statistically significant.

\section{Results}

Gender and age distribution Among the 1137 patients, 940 were male and 197 were female, male: female $=4.771$, age 6 months to 78 years old. Seeing eye trauma can occur in all ages, mainly in the 20-49 age group, accounting for 67.11\%. Occupational distribution Patients with ocular trauma mainly rely on manual workers such as workers and farmers. In addition, preschool children also account for a certain proportion. Injury nature the survey was classified according to clinical habits and was divided into eye contusion, perforation injury, eye appendage trauma and non-mechanical trauma. Table 1 shows the composition of the type of ocular trauma in the five years collected in this survey. Statistical analysis of the difference in composition ratio between adjacent years showed no significant difference $(\chi 2=14.586, P=0.367)$. In addition, it can be seen from Table 1 that the ocular trauma patients who were hospitalized in our hospital were mainly ocular contusion and perforation injury, and their composition ratio was $44.47 \%$ and $40.06 \%$. Complications The complications caused by ocular trauma are complex and diverse. Table 2 summarizes the incidence of complications in patients with ocular trauma during the 5-year period collected in this study. It is known that there are traumatic cataracts, anterior chamber hemorrhage and uveitis. Prognosis Vision and Efficacy Tables $3 \& 4$ show the prognostic visual acuity and efficacy of ocular trauma patients hospitalized in our hospital. A comprehensive comparison of the visual acuity after treatment of the various types of ocular trauma described in the two tables showed significant differences between the groups $(\chi 2=203.476, \mathrm{P}$ $=0.000$ ). The comprehensive analysis of the two tables also clearly showed the difference in the blindness rate of various types of ocular trauma: the blindness rate of the eye piercing injury was the highest (50.08\%), and the blindness rate of the eye appendage was the lowest $(0.77 \%)$.

Table 1: Types of hospitalized patients with ocular trauma in our hospital in 2014/2018 eyes (\%).

\begin{tabular}{|c|c|c|c|c|c|c|}
\hline Eye injury type & \multicolumn{6}{|c|}{ Annual } \\
\hline & $\mathbf{2 0 1 4}$ & $\mathbf{2 0 1 5}$ & $\mathbf{2 0 1 6}$ & $\mathbf{2 0 1 7}$ & $\mathbf{2 0 1 8}$ & Total \\
\hline Eye contusion & $133(43.18)$ & $153(45.54)$ & $146(44.65)$ & $135(45.00)$ & $149(43.95)$ & $716(44.47)$ \\
\hline Perforation injury & $115(37.34)$ & $135(40.18)$ & $134(40.98)$ & $124(41.33)$ & $137(40.41)$ & $645(40.06)$ \\
\hline Eye appendage trauma & $37(12.01)$ & $27(8.03)$ & $24(7.34)$ & $21(7.00)$ & $28(8.26)$ & $137(8.51)$ \\
\hline Non-mechanical injury & $23(7.47)$ & $21(6.25)$ & $23(7.03)$ & $20(6.67)$ & $25(7.38)$ & $112(6.96)$ \\
\hline Total & $308(100)$ & $336(100)$ & $327(100)$ & $300(100)$ & $339(100)$ & $1610(100)$ \\
\hline
\end{tabular}

Table 2: Statistics of complications of ocular trauma patients in our hospital in 2014/2018.

\begin{tabular}{|c|c|}
\hline Complications & Eyes $(\%)$ \\
\hline Traumatic cataract & $611(37.95)$ \\
\hline Anterior chamber hemorrhage & $523(32.48)$ \\
\hline Uveitis & $386(23.98)$ \\
\hline Eye content out & $326(20.25)$ \\
\hline Corneal leukoplakia & $102(6.34)$ \\
\hline Corneal foreign body & $84(5.22)$ \\
\hline Lens out & $47(2.92)$ \\
\hline Iris laceration & $136(8.45)$ \\
\hline Pupil sphincter paralysis & $134(8.32)$ \\
\hline Vitreous blood & $213(13.23)$ \\
\hline Retinal bleeding & $45(2.80)$ \\
\hline Retinal detachment & $15(0.93)$ \\
\hline Secondary glaucoma & $86(5.34)$ \\
\hline
\end{tabular}


Table 3: Statistics of prognosis of hospitalized patients with contusion and perforation in 2014/2018 eyes (\%).

\begin{tabular}{|c|c|c|c|c|c|c|}
\hline Annual & \multicolumn{3}{|c|}{ Eye contusion } & \multicolumn{3}{c|}{ Perforation } \\
\hline & Blind & Low vision & $\geq 0.3$ & Blind & Low vision & $\geq 0.3$ \\
\hline 2014 & $55(41.35)$ & $18(13.53)$ & $60(45.12)$ & $65(56.52)$ & $23(20.00)$ & $27(23.48)$ \\
\hline 2015 & $59(38.56)$ & $22(14.38)$ & $72(47.06)$ & $70(51.85)$ & $25(18.52)$ & $40(29.63)$ \\
\hline 2016 & $52(35.62)$ & $18(12.33)$ & $76(52.05)$ & $67(50.00)$ & $22(16.42)$ & $45(33.58)$ \\
\hline 2017 & $53(39.26)$ & $23(17.04)$ & $59(43.70)$ & $60(48.39)$ & $23(18.54)$ & $41(33.06)$ \\
\hline 2018 & $55(36.91)$ & $27(18.12)$ & $67(44.97)$ & $61(44.53)$ & $31(22.62)$ & $45(32.85)$ \\
\hline Total & $274(38.27)$ & $108(15.08)$ & $334(46.65)$ & $323(50.08)$ & $124(19.22)$ & $198(30.70)$ \\
\hline
\end{tabular}

Table 4: Statistics of prognosis of hospitalized patients with eye appendage trauma and non-mechanical trauma of the eye in $2014 / 2018$ eyes (\%).

\begin{tabular}{|c|c|c|c|c|c|c|}
\hline \multirow[t]{2}{*}{ Annual } & \multicolumn{3}{|c|}{ Eye Appendage Trauma } & \multicolumn{3}{|c|}{ Non-Mechanical Trauma } \\
\hline & Blind & Low vision & $\geq 0.3$ & Blind & Blind & $\geq 0.3$ \\
\hline 2014 & 0 & $4(10.81)$ & $33(89.19)$ & 4(17.39) & $6(26.09)$ & $13(56.52)$ \\
\hline 2015 & 0 & $4(14.81)$ & $23(85.19)$ & $4(19.05)$ & $7(33.33)$ & $10(47.62)$ \\
\hline 2016 & 0 & $1(4.17)$ & $23(95.83)$ & $7(30.43)$ & $5(21.74)$ & $11(47.83)$ \\
\hline 2017 & $1(4.76)$ & 4(19.05) & $16(76.19)$ & $6(30.00)$ & $6(30.00)$ & $8(40.00)$ \\
\hline 2018 & 0 & $4(14.29)$ & $24(85.71)$ & $5(20.00)$ & $8(32.00)$ & $12(48.00)$ \\
\hline Total & $1(0.73)$ & $17(12.41)$ & $119(86.86)$ & $26(23.22)$ & $32(28.57)$ & $54(48.21)$ \\
\hline
\end{tabular}

\section{Discussion}

Eye trauma is a common disease in ophthalmology, and it is also a frequently occurring disease. It accounts for a certain proportion of hospitalized inpatients. With the advancement of modern science and technology, the development of industrialization, and the transformation of transportation, the number of patients suffering from ocular trauma, the number of patients, and the causes of illness are constantly changing. According to statistics, there are 2.5 million new cases of ocular trauma in the United States each year, 4 to 60,000 blindness; 400 cases of ocular trauma per 100,000 people, $40 \%$ of single eye blindness is caused by trauma [3]. In 1988, after the United States Eyes Injury Registry (USEIR) was established in the United States, 15 countries have established similar registration systems [2]. The development of this system allows us to more clearly understand and prevent the cause of ocular trauma, and to master the pattern of ocular trauma, which will help us ultimately improve the clinical treatment effect. However, unfortunately, there is currently no similar regulatory system in China. In recent years, although Chinese scholars have a lot of epidemiological studies on ocular trauma, the research model and classification methods still lack uniform standards, which leads us to lack an overall understanding of the epidemiological characteristics of ocular trauma in the country. In the Chinese northeastern region, Ma Lijun[4], Ren Xiuqing et al. [5], Zhang Fanyou et al. [6] have done eye injury investigations in the corresponding areas, but there are very few surveys on this aspect in western Hubei province. As a comprehensive large hospital, our hospital enjoys a certain reputation in western Hubei province, and the number of patients is large. Therefore, this survey will help to improve the epidemiological data of ocular trauma in this area.

Gender and age analysis this survey clearly indicated that there were significantly more males than females in the ocular trauma patients in our hospital, the ratio of which was 4.77: 1, which is in the statistical range of Xu Jianfeng et al. [7] ( $2.75 \sim 10$ ): 1 inside. This may be significantly more likely than men to be exposed to risk factors for injury. In addition, we also found in the statistics that the causes of eye trauma in male and female patients are also very different: males have more perforation of eyeballs in work or labor, and female eye injuries are mostly caused by eyeball rupture caused by low vision falls. Caused. Except for gender, the obvious age distribution difference of patients with ocular trauma is also a feature we cannot ignore. Li Shuzhen [8] had conducted an epidemiological survey of 1226 hospitalized patients with ocular trauma. The results suggest that the most ocular trauma patients in the 10 to 40 age group. The results of this survey also indicated that $67.11 \%$ of patients with ocular trauma in the $20-49$ age group had no significant difference with most epidemiological findings. It can be seen that the patients with ocular trauma are mainly concentrated in the middle-aged and middle-aged population. This may be because most of the people in this age group are engaged in production labor and have more opportunities to contact the risk factors in the environment such as work and transportation. At the same time, this result warns us to pay more attention to the prevention and treatment of ocular trauma, because the ocular 
trauma patients are the main labor force of the society, not only will cause personal injury to the patients, but also the family economy. Income and social production bring immeasurable losses.

Occupational Distribution Analysis Woo et al. [9] conducted a prospective study of hospitalized patients with ocular trauma at National University Hospital (NUH), which showed that construction workers accounted for $38.3 \%$ of ocular trauma patients. In this survey, the occupational distribution of ocular trauma patients was up to $33.07 \%$. This may be related to the fact that workers have more exposure to risk factors in society. In addition, the survey results also suggest that the number of eye injuries among farmers is second only to workers (32.98\%). We analyze that this may be related to the special national conditions of our country, in addition to the fact that farmers are more engaged in physical labor. In recent years, with the advancement of agricultural production methods, the increase in urban demand for labor, and the increase in the wages of migrant workers, the number of migrant workers working in cities has increased significantly.

However, the risk factor associated with it is that most industrial enterprises, especially private enterprises, lack standardized pre-employment training for migrant workers; in addition, most migrant workers have relatively low cultural level and are attracted by high salaries. They will ignore the security risks. The hospital in this investigation is located in Yichang city, the second largest city in Hubei Province. The local population density is at a medium level in Hubei Province and the local industry is developed, which greatly increases the injury rate of ocular trauma. Thus, combined with the characteristics of the population described in this paper, we have analyzed that in order to reduce the rate of injury caused by ocular trauma, in addition to improving the safety index of equipment and equipment, enterprises are also required to conduct regular preemployment training for workers, especially migrant workers.

Analysis of the nature of injury According to the classification of international eye injuries, ocular trauma can be divided into open and closed eye injuries. Among them, open eye trauma includes:

a) Penetrating through the wound, that is, penetrating the wall of the eye

b) Perforating injury, that is, both the entrance and the exit

c) Foreign matter in the eye

d) Rupture of the eyeball [10]

Closed eye trauma is mainly blunt contusion. Many domestic reports are classified into causes of injury and can be classified into mechanical and non-mechanical ocular trauma. The survey was classified according to clinical habits, and the results showed that the eye contusion was the most; the second was the perforation injury. This is basically consistent with the statistics of Xu Jianfeng et al. [7]. In the statistical process, we found that the cause of eye injury in our hospital is metal injury, boxing, car accident, chemical injury, which may be because the main patient group of eye trauma is young and middle-aged, they are more Participation in production activities, the probability of contact with metal utensils, chemical products; and young people are mostly irritating and eager to love dangerous vehicles such as motorcycles. In addition, we also found that during the Chinese New Year, the number of patients with ocular trauma in our hospital often has a small peak.

We analyzed that this may be related to the traditional Chinese holiday habit of setting off fireworks and firecrackers. However, most of the eye injuries caused by fireworks and firecrackers are compound injuries. In addition to the mechanical damage caused by the explosion shock wave, there may be thermal damage, chemical damage and mechanical damage caused by the explosive itself [11]. Therefore, the degree of injury may be more serious than the general eye injury, and the prognosis is worse. Therefore, we emphasize that ophthalmologists should pay sufficient attention to fireworks and explosions, and if necessary, actively cooperate with other departments to assist in treatment. In addition, the relevant departments of the society must not neglect to carry out corresponding health education on the fireworks and firecrackers during the holiday period to prevent problems before they occur. Eye trauma complications and prognosis visual acuity There are many complications of ocular trauma. Xu Fang [12] statistics show that the most common is uveitis, followed by anterior chamber hemorrhage and traumatic cataract.

This is inconsistent with the results of our survey, which may be easily covered by other signs in the early stage of trauma, and it is also related to the early neglect of clinicians. According to the World Health Organization (WHO) standards for low vision and blindness: the best corrected visual acuity of better eyes is $\geq 0$. 05 , and $<0.3$ for low vision; better corrected visual acuity of better eye $<0.05$ is blind [13]. This investigation investigated the prognosis of eye adnexal trauma with good visual acuity, followed by nonmechanical trauma. Sympathetic ophthalmia can be caused by perforation injury; eye contusion is often accompanied by damage of lens, retina, etc., and the prognosis of both is poor. Based on the survey data in Tables 3 and 4, we can also see that the perforation injury has the highest blindness rate (50.08\%), followed by eye contusion (38.27\%). In addition, there is a phenomenon worthy of our attention in Tables $3 \& 4$. That is, the eye injury caused by any cause has been reduced in recent years. We have analyzed the possibility of timely treatment of patients with ocular trauma. And in recent years, advances in ophthalmic microsurgery technology have been related to continuous improvement of medical devices.

Treatment and prevention Because the structure of the eye is fine and complex, we should emphasize timely and reasonable treatment in the event of ocular trauma. We agree that there is a clear correlation between the time of visit and the rate of blindness. Because prolonged visit time may increase the chance of infection and wound edema will increase the difficulty of treatment and 
is also very unfavorable for wound recovery. In this regard, Jing Chunxia et al. [14] had a single-factor Logistic regression analysis of treatment timing and prognosis, and found that the longer the time from surgery, the longer the operation time and the longer the hospital stay, the worse the prognosis. Emergency treatment of ocular trauma should be based on the type of trauma. Eye blunt contusion should be based on conservative treatment, and actively take measures such as anti-inflammatory, hemostasis, and absorption. For eye-piercing injuries, we recommend early closure of the wound, supplemented by anti-inflammatory, hemostasis and other treatments. For unrestrained ocular rupture, early eyeball removal should be performed. Complex ocular trauma is often accompanied by a variety of eye injuries, especially accompanying complications of ocular trauma may cause greater harm to patients.

For the most common complication of traumatic cataract in this survey, it has been advocated that conservative treatment should be taken unless the lens cortex enters the anterior chamber or secondary glaucoma, and surgery is performed 3 months or more after the injury. However, through clinical observations in recent years, it has been found that in the months after the injury, the anterior lens capsule may form a mechanical membrane and may adhere to the iris, which will increase the difficulty of cataract surgery, increase the incidence of surgical complications, and even lose. Surgery opportunities. Therefore, most people now advocate cataract extraction 10 days after injury [15]. It is worth mentioning that in recent years, the level of microsurgical techniques such as vitrectomy surgery in China has been continuously improved, and the corresponding surgical instruments and materials have been continuously improved, and the efficacy of ocular trauma has also been greatly improved. As mentioned above, the group of patients with ocular trauma is mainly young and middle-aged, and the causes of injury are mostly risk factors in work and traffic environment. From this we can see that most eye injuries are preventable.

We should continue to strengthen the publicity of eyesight hygiene common sense; conduct pre-employment training for new workers and migrant workers; obey traffic rules, reduce traffic accidents; and strengthen protection for preschool children. In the event of ocular trauma, early delivery to the doctor for standardized treatment, try to improve the prognosis vision and reduce the blindness rate. In addition, although there are many studies on ocular trauma in China, the lack of unified statistics and classification methods has led to difficulties in communication and difficulty between different results. We expect the corresponding consensus to be raised as soon as possible.

\section{References}

1. Roodhooft JM (2000) Leading causes of blindness worldwide. Bull Soc Belge Ophthalmol 283: 19-25.

2. May DR, Kuhn FP, Morris RE (2000) The epidemiology of serious eye Injuries from the United States Eye Injury Registry. Graefes Arch Clin Exp Ophthalmol 238(2): 153-157.

3. Hui Yannian (2000) The prevention and treatment of ocular trauma should be given great attention. Chinese Trauma Journal 16(7): 389-390.

4. Ma Lijun (1997) 1737 cases of ocular trauma analysis report. Journal of Ocular Trauma Occupational Eye Disease 19(1): 38-39.

5. Ren Xiuqing, Li Guozhuang, Zhang Zuojun (1998) Statistical analysis of 270 cases of hospitalized patients with ocular trauma. Ocular trauma occupational eye disease journal 20(6): 587-588.

6. Zhang Fanyou, Zhang Hui, Li Xuhong (2001) Investigation and analysis of ocular trauma cases in Dalian. Chinese Journal of Practical Ophthalmology 19(4): 307-308.

7. Xu Jianfeng, Wang Yusheng (2004) Epidemiological status of ocular trauma in mainland China. International Journal of Ophthalmology 4(6): 1069-1076.

8. Li Shuzhen (2001) Analysis of related factors of thousands of eye injuries. Journal of Ocular Trauma Occupational Eye Disease 23(1): 20-21.

9. Woo JH, Sundar G (2006) Eye injuries in singapore - Don't risk it. Do more. A prospective Study. Ann Acad Med Singapore 35(10): 706-718.

10. Kuhn F, Morris R, Witherspoon D(1996) A standardized classification of ocular trauma. Ophthalmology 103(2): 240- 243.

11. Wei, Li Shengyun, Zhang Guiju (2011) 142 cases of ocular trauma caused by fireworks and firecrackers. Chinese Journal of Ocular Trauma Occupational Eye Disease 33(2): 148-149.

12. Xu Fang (2011) Analysis of related factors of ocular trauma. Journal of Clinical and Experimental Medicine 10(17): 1377-1378.

13. World Health Organization. Internation statistical classification of diseases and related health problem. Tenth revision Geneva: World Health Organization.

14. Jing Chunxia, Chi Guibo, Wu Chipeng (2003) Analysis of risk factors affecting visual acuity of ocular trauma. Journal of Ocular Trauma Occupational Eye Disease 25(3): 154-155.

15. Xu strengthens, Guo Jingjing (2003) Treatment of 203 cases of ocular trauma and its complications. Harbin Medical 23(3): 30-31.

\section{ISSN: 2574-1241}

DOI: 10.26717/BJSTR.2019.23.003949

Liang Liang. Biomed J Sci \& Tech Res

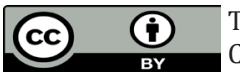

This work is licensed under Creative Commons Attribution 4.0 License

Submission Link: https://biomedres.us/submit-manuscript.php

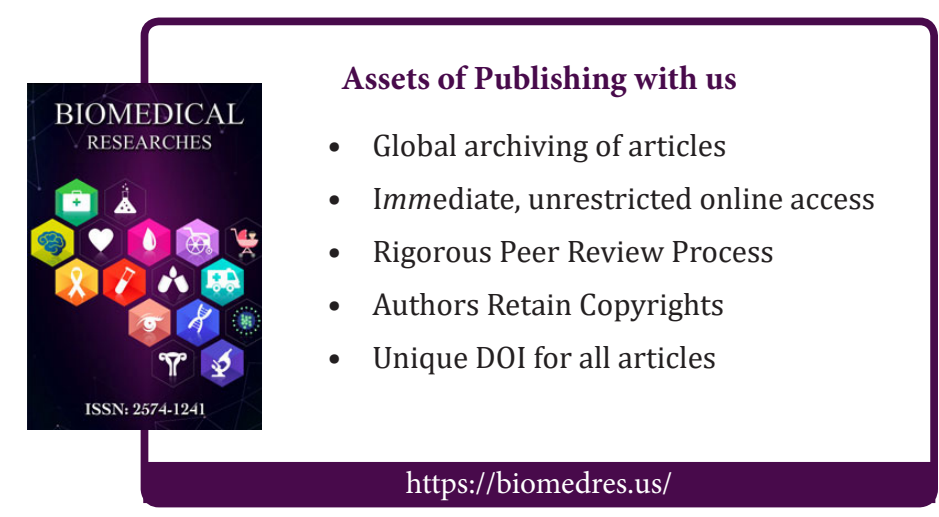

